\title{
A Heuristic Approach for the Vertex Cover Problem
}

\author{
Omar Kettani, Faycal Ramdani, Benaissa Tadili \\ LPG Lab \\ Scientific Institute \\ Mohamed V University, Rabat
}

\begin{abstract}
A vertex cover is a subset of the vertex set of a given graph $G$ such that every edge in $\mathrm{G}$ has at least one endpoint in this set. The Minimum Vertex Cover problem consists to find the minimum sized vertex cover in a graph. This problem which belongs to the class of NP-hard graph theoretical problems, has many practical applications in various fields. In this article, we propose a novel heuristic algorithm for solving this problem. The test results obtained on some graph examples available in the literature confirm the effectiveness of the proposed method.
\end{abstract}

\section{Keywords}

Minimum Vertex Cover problem, heuristic algorithm

\section{INTRODUCTION}

Given an undirected graph $\mathrm{G}=(\mathrm{V}, \mathrm{E})$, a vertex cover is a subset $\mathrm{C} \subseteq \mathrm{V}$, such that every edge in $\mathrm{G}$ has at least one endpoint in C. The Minimum Vertex Cover problem is to find the minimum sized vertex cover in a graph. The decision version of this problem is one of Karp's 21 NP-complete problems [1]. This problem has many practical applications, including: VLSI design [2], multiple sequence alignments for computational biochemistry [3], network security and scheduling.

Several heuristics and approximations algorithms have been proposed in solving the Minimum Vertex Cover problem. Chleb [4] proposed a branch-and-bound algorithm in finding near optimal solutions which explores the configuration space by deciding about the presence or not of one node by the cover in each step of the recursion and recursively solving the problem of the remaining nodes. Covered node and all adjacent edges are removed, while an ignored node remains, but cannot be selected in deeper levels of the recursion. Friedrich [5], shows the cases for which the solutions obtained by two approximation algorithms can be improved by an evolutionary algorithm. Clarkson [6] proposed the following improved greedy algorithm:

Input: a graph $\mathrm{G}=(\mathrm{V}, \mathrm{E})$

Output: a vertex cover $\mathrm{C}$

$\mathrm{C} \leftarrow \varnothing$

while $\mathrm{E} \neq \varnothing$ current graph

choose vertex $\mathrm{v} \in \mathrm{V}$ of maximum degree in the

$$
\begin{aligned}
& \mathrm{C} \leftarrow \mathrm{C} \square\{\mathrm{v}\} \\
& \mathrm{E} \leftarrow \mathrm{E} \backslash\{\mathrm{e} \in \mathrm{E}: \mathrm{v} \in \mathrm{e}\}
\end{aligned}
$$

end while

return $\mathrm{C}$

Recently, Alom [7] solved this problem by introducing an $\mathrm{O}(|\mathrm{E}|)$ greedy algorithm for this problem. This algorithm selects the vertex which has maximum number of edges incident to it. All the edges are discarded incident to that vertex. If more than one vertex have the same maximum number of edges, this algorithm select the vertex which have at least one edge that is not covered by other vertices. This process is repeated until to cover all vertices.

As a preliminary work, this paper describes an alternative heuristic algorithm and to tests its performance on some examples.

The rest of this paper is organized as follows: In section II we describe the proposed approach for solving this problem, whereas section III reports the results of the application of the method on some graph examples taken from the literature. Finally, conclusions are given in section IV.

\section{THE PROPOSED APPROACH}

Given an undirected graph $G=(V, E)$ with vertex set $V(G)$ of cardinality $|\mathrm{V}(\mathrm{G})|=\mathrm{n}$, and edge set $\mathrm{E}=\mathrm{E}(\mathrm{G})$ of cardinality $|\mathrm{E}(\mathrm{G})|=\mathrm{m}$.

The neighborhood of a vertex $\mathrm{v} \in \mathrm{V}$ is the $\operatorname{set} \mathrm{N}(\mathrm{v})=\{\mathrm{u} \in \mathrm{V}$ : vu $\in \mathrm{E}\}$.

The main idea of the method consists to start with an initial vertex $\mathrm{v} \in \mathrm{V}$ of minimum degree, and to set the initial cover $\mathrm{C}$ as the neighborhood $\mathrm{N}(\mathrm{v})$ of $\mathrm{v}$. Then we search for another vertex $w \in V$ not yet considered which minimize $|N(w) \cup C|$, then $C$ is updated by $N(w) \cup C$. This process is repeated until all vertices have been scanned and resulting $C$ is returned. We summarize the pseudo code of the proposed algorithm as follows:

Input: $A$ Graph $G=(\mathrm{V}, \mathrm{E})$

Output: A Vertex Cover C

choose a vertex $\mathrm{v} \in \mathrm{V}$ of minimum degree

$$
\begin{aligned}
& \mathrm{C} \leftarrow \mathrm{N}(\mathrm{v}) \\
& \mathrm{I} \leftarrow\{\mathrm{v}\} \\
& \text { while } \mathrm{V} \backslash(\mathrm{C} \square \mathrm{I}) \neq \varnothing \\
& \text { choose a vertex } \mathrm{w} \in \mathrm{V} \backslash \\
& \text { minimum } \\
& \qquad \mathrm{C} \leftarrow \mathrm{N}(\mathrm{w}) \square \mathrm{C} \\
& \mathrm{I} \leftarrow\{\mathrm{w}\} \square \square \mathrm{I}
\end{aligned}
$$$$
\text { choose a vertex w } \in \mathrm{V} \backslash(\mathrm{C} \square \mathrm{I}) \text { such that }|\mathrm{N}(\mathrm{w}) \quad \square \mathrm{C}| \text { is }
$$

end while

return $\mathrm{C}$

Complexity analysis of the algorithm:

Since the number of iterations of while loop is at most $n=\mid V$ $(\mathrm{G}) \mid$, and $|\mathrm{V} \backslash(\mathrm{C} \cup \mathrm{I})|$ is bounded by $\mathrm{n}$. So the time complexity of this algorithm is $\mathrm{O}\left(|\mathrm{V}|^{2}\right)$. 


\section{EXAMPLES}

In order to assess the effectiveness of this approach, we have tested this method on some graph examples taken from the literature. Results are reported in the following table:

Table 1 Some graph examples used for testing the proposed method.

\begin{tabular}{|c|c|c|}
\hline Graph & $|\mathbf{V}|$ & $|\mathbf{C}|$ \\
\hline Tetrahedron & 4 & 3 \\
\hline Kuratowski [8] & 6 & 3 \\
\hline Octahedron & 6 & 4 \\
\hline Bondy-Murty G1[9] & 7 & 4 \\
\hline Wheel graph W8 & 8 & 5 \\
\hline Cube & 8 & 4 \\
\hline Petersen [14] & 10 & 6 \\
\hline Bondy-Murty G2[9] & 11 & 7 \\
\hline Grötzsch [10] & 11 & 6 \\
\hline Herschel [11] & 11 & 5 \\
\hline Icosahedron & 12 & 9 \\
\hline Bondy-Murty G3 [9] & 14 & 7 \\
\hline Bondy-Murty G4 [9] & 16 & 7 \\
\hline Ramsey graph [12] & 17 & 14 \\
\hline Folkman [13] & 20 & 10 \\
\hline Dodecahedron & 20 & 12 \\
\hline Tutte-Coxeter [14] & 30 & 15 \\
\hline Thomassen [15] & 34 & 20 \\
\hline
\end{tabular}

The following figures represent some tested graphs, where black vertex belongs to the cover sets found by the proposed method.

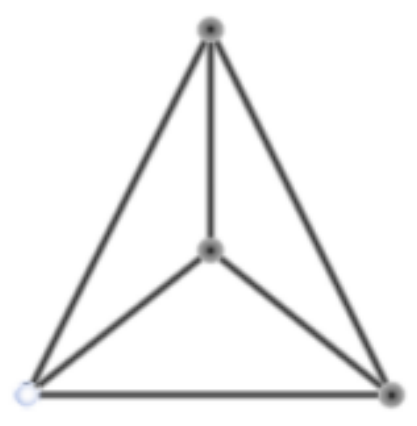

Figure 1: The Tetrahedron

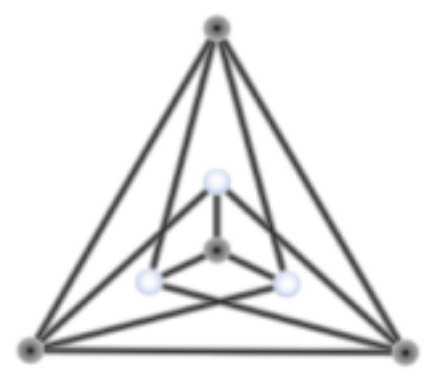

Figure 2: The Bondy-Murty graph G1

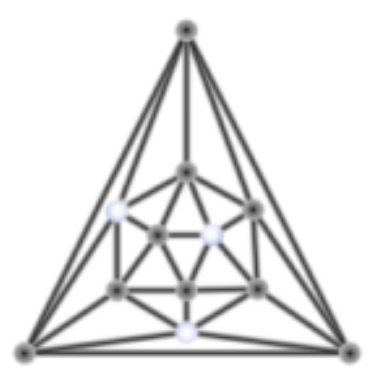

Figure 3: The Icosahedron

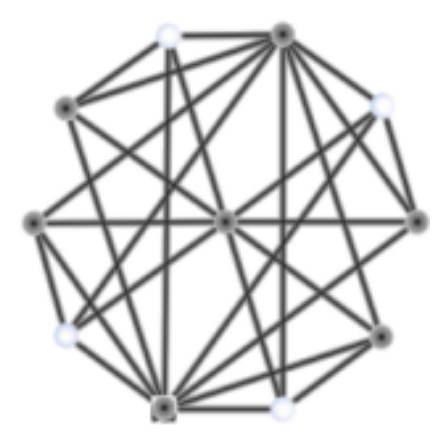

Figure 4: The Bondy-Murty graph G2 


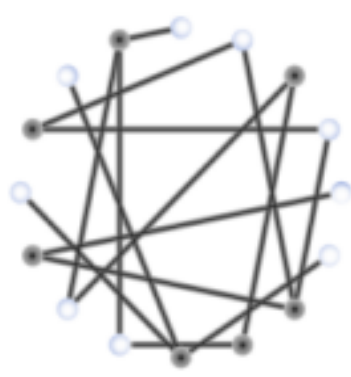

Figure 5: The Bondy-Murty graph G4

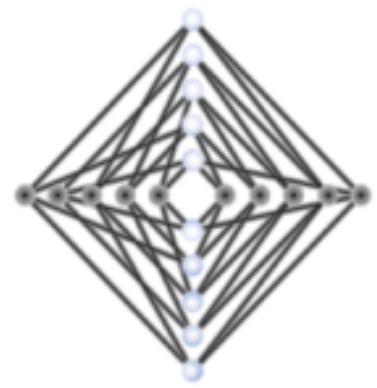

Figure 6: The Folkman graph

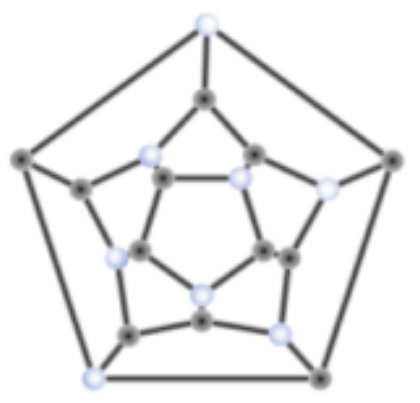

Figure 7: The Dodecahedron

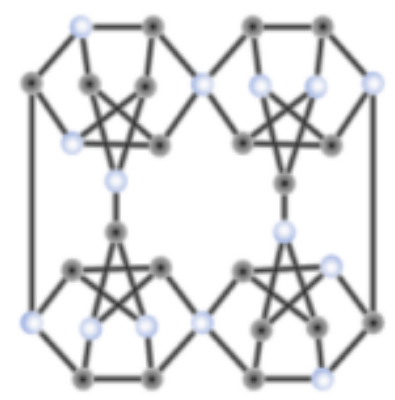

Figure 8: The Thomassen graph

\section{CONCLUSION}

In this article, we have suggested a novel heuristic algorithm in order to solve the Minimum Vertex Cover problem. We have tested this method on some graph examples taken from the literature. Results show that the proposed approach is useful. Interesting challenge is to associate this method with some meta-heuristic search and to test the resulted hybrid algorithm on large graph instances. In forthcoming work, we will also investigate whether this method may be applied to other related graph optimization problems, like the maximum clique problem.

\section{REFERENCES}

[1] Karp R. M., "Reducibility Among Combinatorial Problems" . In R. E. Miller and J. W. Thatcher (editors). Complexity of Computer Computations. New York: Plenum. pp. 85-103.

[2] Cheetham J., Dehne F., Rau-Chaplin A., Stege U., Taillon ,P. "Solving large FPT problems on coarse grained parallel machines" Journal of Computer and System Sciences 67 (4) (2003) 691-706.

[3] Gomes, F.C., Meneses, C.N., Pardalos, P.M., Viana, G.V.R "Experimental analysis of approximation algorithms for the vertex cover and set covering problems" Computers and Operations Research 33(12), 3520-3534 (2006).

[4] Chleb M. and Chleb J., "Crown reductions for the Minimum Weighted Vertex Cover problem" Electronic Colloquium on Computational Complexity, Report No. 101 (2004)

[5] Friedrich T. He J., "Analyses of Simple Hybrid Algorithms for the Vertex Cover Problem" Evolutionary Computation. MIT Press 2009.

[6] Clarkson K., "A modification to the greedy algorithm for the vertex cover problem" IPL, vol 16:23-25,(1983).

[7] Alom B. M., Das S. and Abdur Rouf M. "Performance Evaluation of Vertex Cover and Set Cover Problem using Optimal Algorithm" . DUET Journal Vol. 1, Issue 2, June 2011.

[8] Kuratowski K., "Sur le problème des courbes gauches en topologie," Fund. Math.,1930.

[9] Bondy J.A. and Murty U.S.R. , "Graph Theory with Applications," Elsevier Science Publishing Co., Inc, 1976.

[10] Grötzsch H., "Ein Dreifarbensatz für dreikreisfreie Netz auf der Kugel,” Z. Martin-Luther-Univ., 1958.

[11] Herschel A.S., Sir Wm., "Hamilton's Icosian Game," Quart. J. Pure Applied Math.

[12] Ramsey F.P. , On a problem of formal logic, Proc. London Math. Soc., 1930.

[13] Folkman J., "Regular line-symmetric graphs," J. Combinatorial Theory, 1967.

[14] Coxeter H.S.M. and Tutte W.T., "The Chords of the Non-Ruled Quadratic in PG(3,3)," Canad. J. Math., 1958.

[15] Thomassen C., Hypohamiltonian and hypotraceable graphs, Discrete Math., 1974. 